\title{
Incisiones transversas curvas en cirugía ginecológica y obstétrica ${ }^{(*)}$
}

\author{
Doctor Carlos Arturo Goubert (**)
}

En este trabajo voy a hacer una revisión sobre el conocido tema de las incisiones transversas en cirugía abdominal, especialmente ginecológica y obstétrica, analizando artículos recientes y antiguos sobre el tema y presentando la estadística de los resultados obtenidos en el Departamento de Ginecología del Hospital de San Juan de Dios, de Bogotá (14) durante el año de 1958 y las obtenidas por un distinguido ginecólogo (2) en su clientela particular.

\section{Historia}

El famoso obstetra francés Baudelocque describió la primera. incisión transversa para practicar cesáreas en 1823, pero otros au. tores remontan las primeras descripciones aún muchos años antes (1). Pfannenstiel en 1900 hizo notar las ventajas de estas incisiones, especialmente de la que lleva su nombre. En 1907 Maylard practica otra incisión transversal, luego Cherney en 1941 la que lleva su nombre; Rodríguez López (1947) y Alvarez Bravo (1951) hacen modificaciones del Pfannenstiel; Williams en 1954, Durán ıen 1955, Greenhill en 1955 y Gómez F. en 1957 se ocupan nuevamente del tema y describen algunas modificaciones, presentando estadísticas que hacen ver las ventajas y los inconvenientes de este tipo de laparotomías.

\section{Consideraciones anatómicas}

No entró a tratar por separado la anatomía de la región, pues no hay nada nuevo que añadir a lo que todos sabemos al respecto,

(*) Trabajo presentado para ingresar como Miembro Afiliado de la Sociedad Colombiana de Obstetricia y Ginecología.

(**) Residente del Departamento de Ginecología y Obstetricia del Hospital de San Juan de Dios, Bogotá, D. E. 
pero haré resaltar los detalles necesarios para comprender determinados aspectos de la técnica.

\section{Técnica}

La incisión de Pfannenstiel modificada por Rodríguez es la siguiente:

Incisión de piel iniciándola dos traveses de dedo por debajo y adentro de la espina ilíaca anterior y superior pasando a ras del borde piloso del monte de Venus, altura que varía según los distintos autores, quienes dan como puntos de refierencia "dos pulgadas por encima del borde superior del púbis", otros dicen "en la unión del tercio inferior con los dos tercios superiores de ia distancia umbílico-suprapubiana", y otros "dos traveses de dedo por encima del borde superior del púbis"; pero como se ve, todas las medidas concuerdan aproximadamente; el otro extremo de la incisión se halla en sitio similar pero del lado opuesto al de la incisión. La incisión es ligeramente cóncava hacia arriba; generalmente corresponde a un pliegue natural de eisa región, por to cual se debe procurar seguirlo para conseguir mejores resultacos estéticos; si existe un abdomen péndulo, debe levantarse con ia mano y pasar por el surco que forma. En profundidad, la inci. sión solo debe llegar ligeramente debajo de la dermis, para evitar cortar vasos gruesos que sangran y obligan a usar pinzas hemostáticas. Se debe profudizar la incisión en la línea media, en un ancho de 4 centímetros, hasta hallar la aponeurosis anterior de los rectos, incidirla a los lados de la línea media formando dos pequeñas ventanas que dan paso a los dedos índices del cirujano y ayudante', con los cuales se hace tracción en sentido lateral para divulsionar la aponeurosis y tejido celular subadyacente. Si se desea, se puede disecar ligeramente el rafé mediano con tijeras. Si no se obtiene buena disección lateral, se debe terminar cortando con tijeras la aponeurosis y divulsionando con los dedos.

Se encuentran los rectos anteriores; se toman los colgajos superior e inferior con pinzas de Rochester y con ayuda de tijeras y disección roma se lleva la separación de los colgajos hasta el ombligo y el púbis. Es importante llegar hasta estos sitios para que la incisión sea suficientemente amplia. Separando los rectos lateralmente; se toma el peritoneo y fascia transversalis con pinzas de Kelly en la parte superior para evitar la vejiga, se abre pe. queño ojal, se reparan los bordes con pinzas de Rochester y luego se abre el peritoneo y fascia transversalis longitudinalmente 
con la técnica habitual, previa colocación de una compresa para evitar lesionar las vísceras. Luego se procede a colocar el separacor abdominal, previa protección de pared con compresa, según las preferencias de cada cual.

Fotografías de la técnica de la incisión de Pfannenstiel modificada

Se muestra una serie de fotografias de la incisión de Pfanxenstiel modificada por Rodríguez y se hace uso de otra modificación introducida por Alvarez. Solo se muestra la técnica de la incisión, pues el cierre está claramente descrito y no tiene nada de especial. No se muestran técnicas del Maylard ni de Cherney por ser suficientemente conocidas. Al final se aprecian los resultados estéticos. 


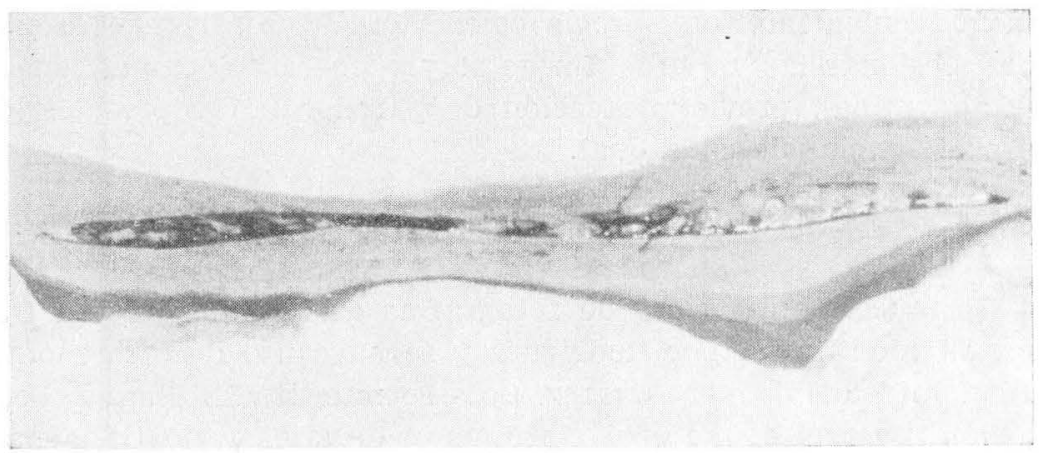

Profundidad de la incisión en dermis: nótese que prácticamente no hay hemorragia a pesar de no haberse colocado pinzas hemostáticas.

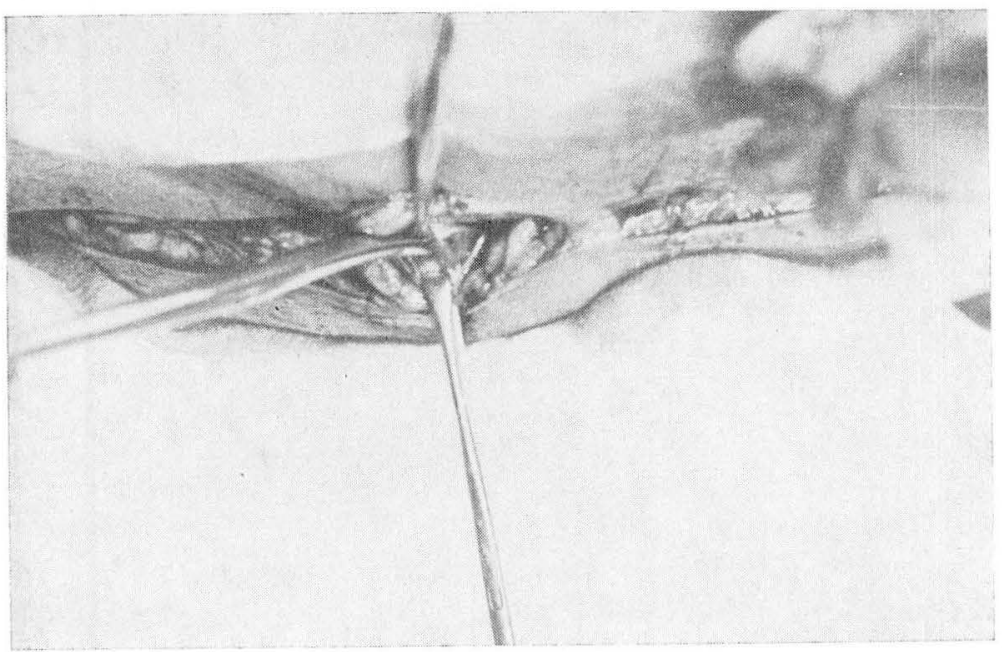

Corte de la aponeurosis: se profundiza en la parte media de la incisión hasta llegar a aponeurosis y se abren dos hojales despegando ligeramente el rafé mediano. 


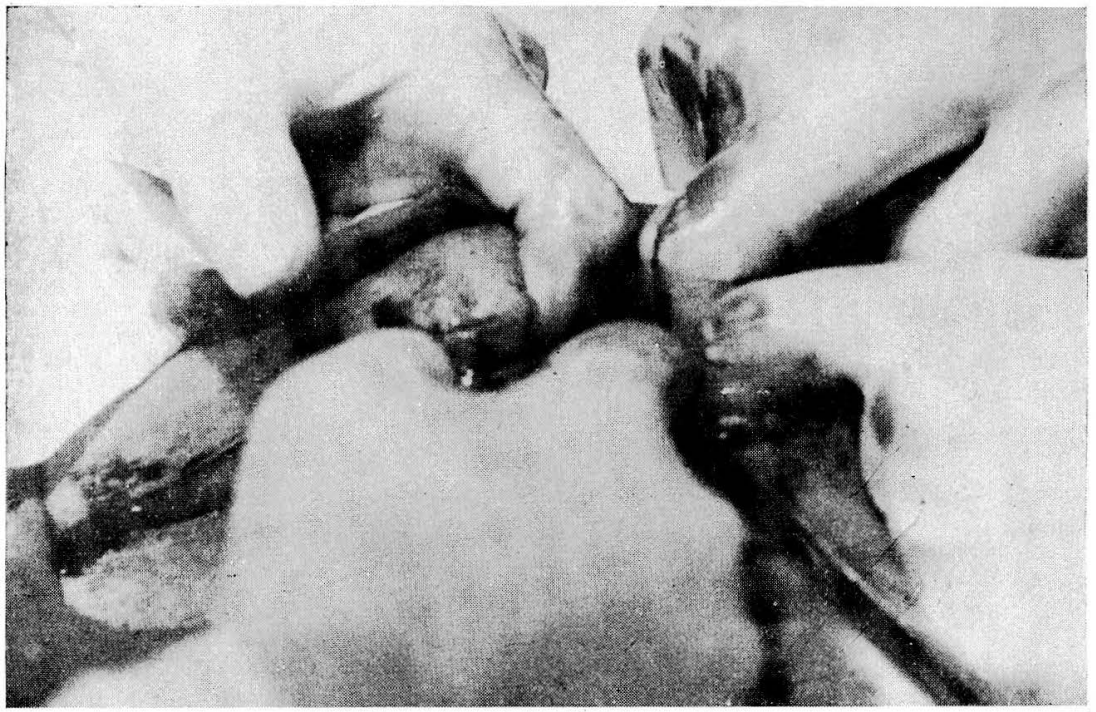

Divulsión de la aponeurosis: introduciendo los dedos índices del cirujano y del ayudante se tracciona lateralmente.

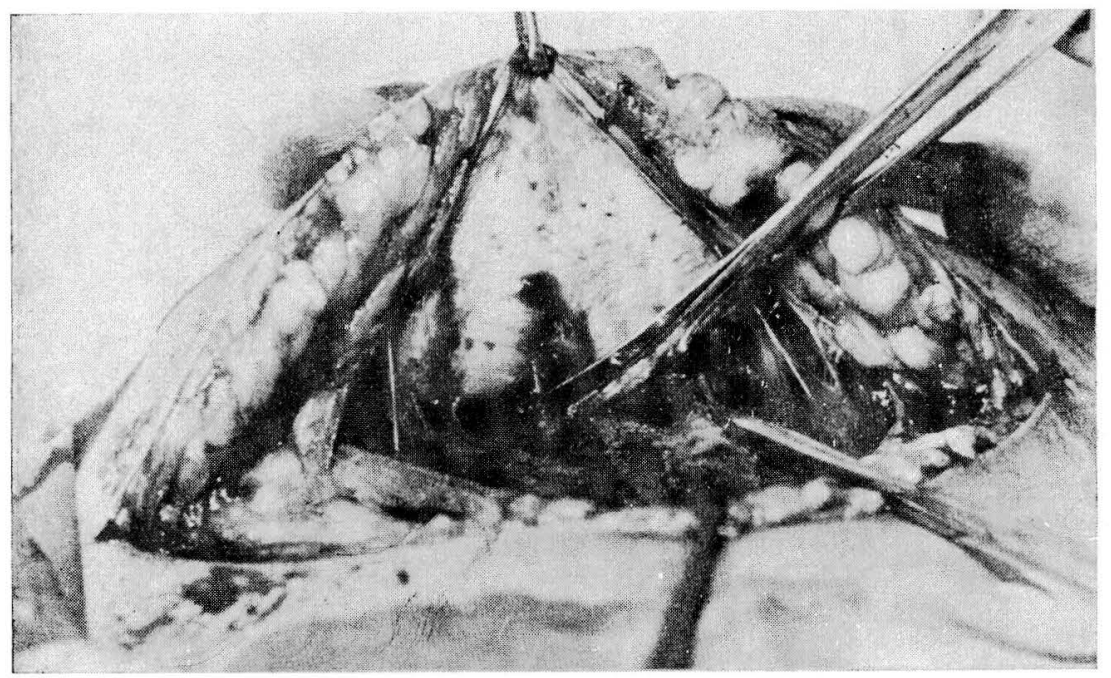

Disección de la aponeurosis: se toman el borde del colgajo superior con un Allis y se desprende en la línea media de la aponeurosis y peritoneo con ayuda de tijeras y de torunda. 


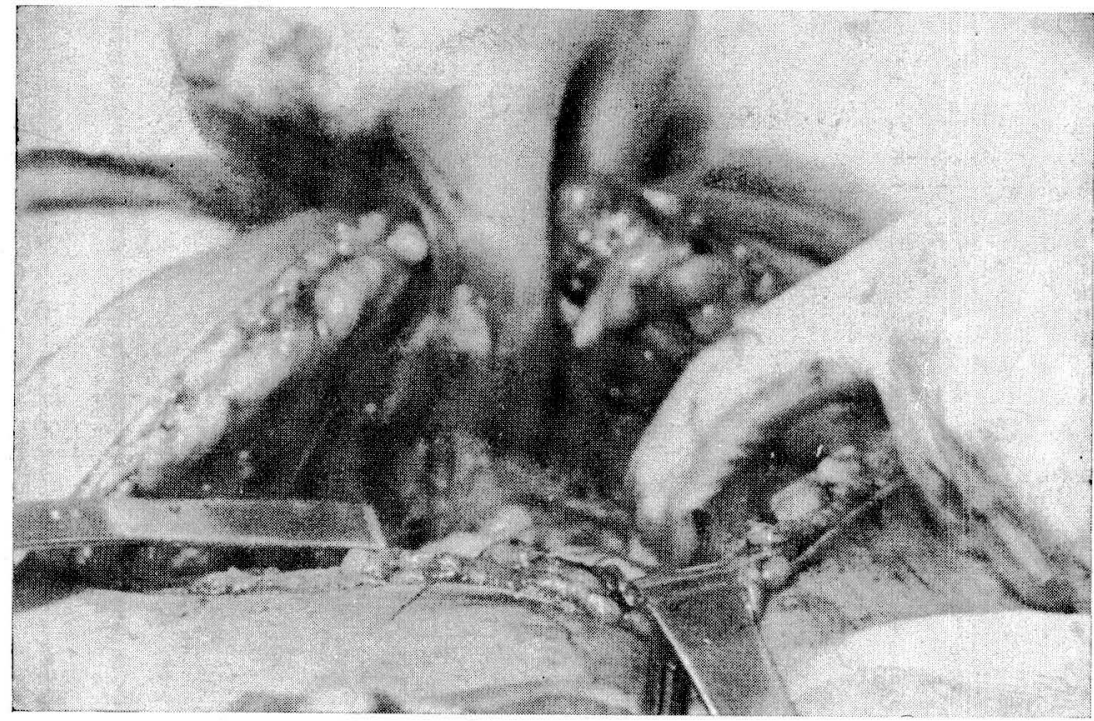

Exposición del peritoneo: en caso de que no sea aparente la línea media por la separación espontánea de los rectos, se tratan de separar con el cabo del bisturí o con una gasa manejada con una pinza de disección sin garra; si esto no es posible, se divulsiona el plano muscular con los dedos como en la técnica usada para una incisión transrectal, tal como se aprecia en esta fotografía.

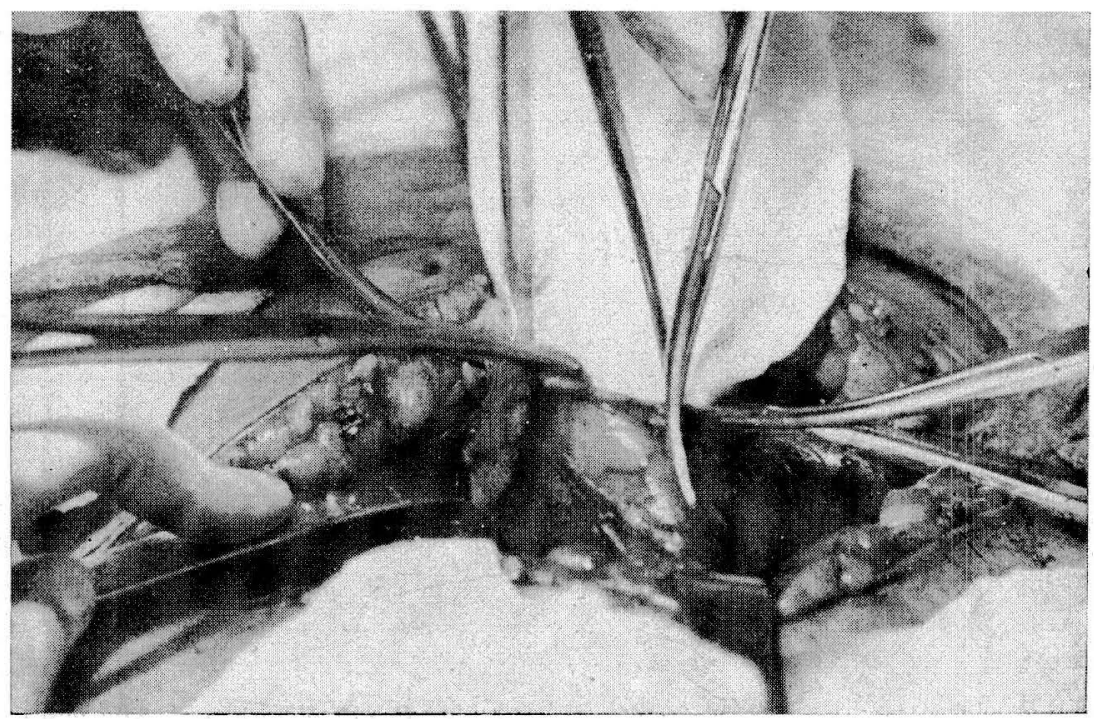

Apertura de peritones: se hace con la técnica habitual en la parte superior de la incisión para evitar lesionar vejiga. 


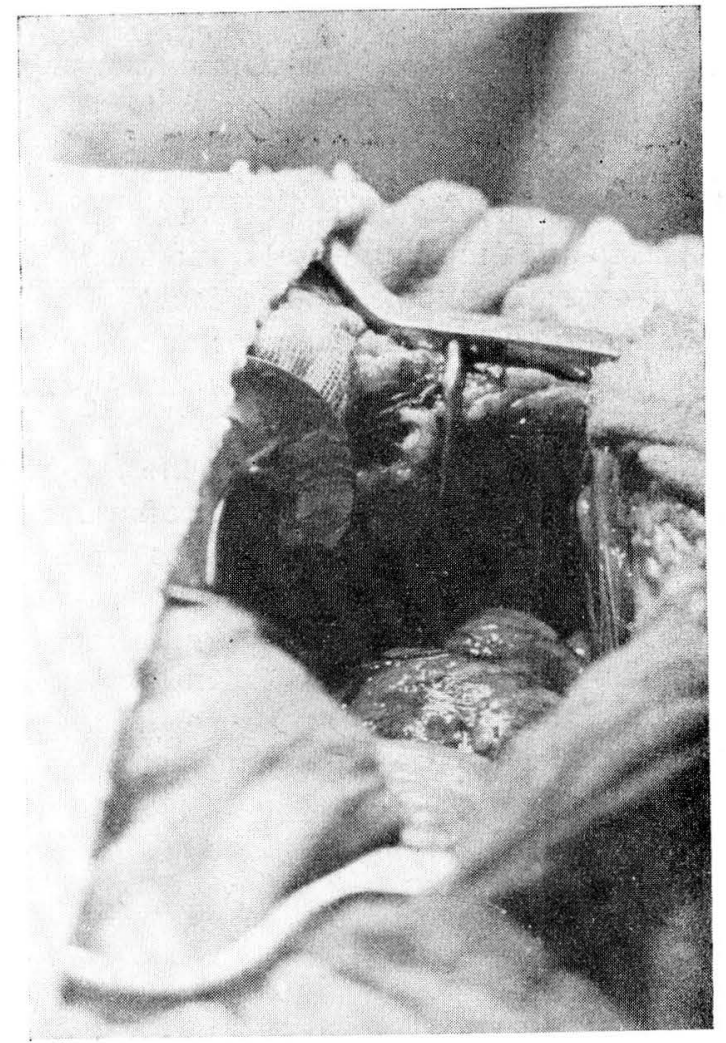

Coloceción del separador: en el Pfannenstiel Rodríguez se coloca el separador en forma similar a la de las laparotomías verticales. En esta fotografía pcdemos observar el magnífico campo dado por la incisión de Pfannenstiel Rodríguez para una histerectomía abdominal total por mionsatosis uterina.

El cierre de esta incisión es relativamente fácil, a pesar de ha_ ber anestesia superficial. Se cierra peritoneo y fascia transversalis en un solo plano con catgut simple o cromado número 1 puntos separados o contínuos; se pasan unos pocos puntos separados de catgut simple para afrontar los bordes internos de los rectos; sí se divulsionaron los músculos anchos se deben aproximar con puntos separados de catgut simple. La aponeurosis se cierra en general con material no absorbible, como seda o algodón puntos separados; se pasan algunos puntos de catgut simple separados por grasa. La piel se puede cerrar con agrafes o seda. 


\section{Modificaciones de esta incisión. Incisión de Pfannenstiel típica.}

En la incisión original el tejido celular subcutáneo es cortado con bisturí y se produce abundante hemorragia que demora la incisión. La aponeurosis es cortada con tijeras o bisturí en sentido transversal. El resto de la incisión es tal como queda descrita antes.

Alvarez Bravo (1) ha modificado la incisión de Pfannenstiel Rodríguez, insistiendo en que se deben cortar los músculos anchos del abdomien y sus aponeurosis si es necesario, para obtener luz suficiente.

Si se trata cle incisiones ien mujeres vírgenes, y se halla dificultad para desgarrar la aponeurosis, propone añadir dos maniobras a la técnica mencionada: 1) pasar una pinza de Rochester o similar entre el tojido celular subcutáneo y la aponeurosis subyacente inmediatamente en contacto con ella y retirarla abierta y luego 2) volver a pasar la pinza de Rochester entre la aponeurosis y el plano muscular y en contacto con la aponeurosis y retirarla igualmente abierta para obtener así una separación entre ios diferentes planos, divulsionándolos con los dedos en la forma descrita pero por separado, primero el plano de tejido celular y después el plano aponeurótico.

\section{Incisión Maylard}

Incisión de piel y tejido celular aubcutáneo como en el Pfannenstiel original, sección transversal de aponeurosis anterior de recto y si es necesario prolongándola seccionando aponeurosis de oblicuos y transverso. Se cortan y ligan generalmente las venas epigástricas superficiales; sección de músculos rectos a la altura de la incisión de aponeurosis, para ello se pasa el dedo índice, o éste y el dedo medio por debajo del músculo y se secciona, debiendo evitarse hacer grandes desprendimientos de músculos de su lecho aponeurótico. En el tercio externo se seccionan y ligan o se separan los vasos epigástricos profundos. Se repara el peritoneo lateralmente a la altura de la incisión y se abre transversalmen. te, con la técnica habitual, un ojal por el cual se pasa el dedo para verificar la altura de la vejiga, de manera que el corte del peritoneo, que es transversal, pase a un través de dedo por encima del borde superior de la vejiga. En la línea media se debe ligar el uraco y los restos embrionarios de las arterias umbilicales, teniendo cuidado de unirlos al efectuar el cierre de la pared. También debe cuidarse si existen divertículos vesicales. Si es necesa- 
rio, lateralmente se desgarran las fibras de los músculos oblícuos. Colocación del separador abdominal autostático con la técnica que acostumbre el cirujano.

El cierre de esta incisión no es laboriosa si se procede en la siguiente forma: flejar ligeramente al paciente si no se logra aproximación satisfactoria y suturar el peritoneo y fascia trans. versalis en un solo plano con catgut simple o cromado; unión de uraco y restos de arterias umbilicales con puntos en U. El cierre del peritoneo debe ser cuidadoso. Se pueden poner algunos puntos de afrontamiento de los bordes internos de los rectos entre sí, y se deben siempre reparar lateralmente los músculos anchos de abdomen así como sus fascias, pero no se debe intentar la unión de los segmentos superior e inferior de los rectos por considerarse difícil, no satisfactorio e innecesario (25), (13), (15), pues al afrontar correctamente las hojas posterior y anterior de ia aponeurosis, los extremos del músculo quedan en contacto, favoreciendo una adecuada cicatrización. La aponeurosis, tejido celular subcutáneo y piel se cierran como en el Pfannenstiel.

\section{Modificaciones a esta técnica}

Esta es una técnica que muchos ginecólogos norteamericanos usan y le dan preferencia sobre otras laparotomías transversas (25), (13), pero los anteriormente citados y otros (26), (27) están de acuerdo en que prácticamente siempre se debe usar la incisión transversa en cirugia ginecológica y obstétrica.

\section{Técnica de Cherney}

La técnica de Cherney difiere esencialmente de la anterior en que los músculos rectos no son cortados en su parte muscular sino en la tendinosa, a unos 5 milímetros del borde superior del pubis, el cual se debe visualizar bien durante la disección. Los piramidales se dejan en la hoja anterior del recto. Se ligan por transfixión gruesa arteria cerca del púbis. El oblicuo menor y el transverso se cortan paralelamente a la arcada crural, a unos 15 milímetros; se rechaza todo el colgajo hacia arriba para poder abrir el peritoneo por encima de la vejiga. Se halla gruesa capa de tejido celulo-grasoso preperitoneal y los vaisos epigástricos que «e ligan. El peritoneo se abre con la técnica vista para el Maylard.

Para el cierre de esta laparotomía lo esencial es la reimplan¿qción de los rectos que se deben suturar al tendón de donde fueron seccionados, pero puede ocurrir que uno de los cabos del tendón, especialmente el superior, esté deshilachado; si esto ocurre 
se lidce una sutura helicoidal en el borbe inferior y luego se pa. san funtos simples; si es menos, se pasan puntos simples pero en la parte media se hacen helicoidales. Si se tiene el problema en el extremo inferior por no hallarlo porque se cortó a ras del púbis, o al suturar se deshilacha, se puede suturar el recto a la vaina Ge los rectos pasando puntos perforantes a través de ésta y pirarnłdales si existen y anudándolos por delante de la aponeurosis o suturar el tendón al piramidal. El resto de la reconstrucción se race con la técnica vista en el Maylard.

Algunos autores, especialmente argentinos, opinan (9) que es. ta incisión es superior a aquellas que seccionan cuerpo muscular, pero parece tener más seguidores el Maylard (25), (13), principalmente ers la escuela norteamericana.

\section{Otras incisiones transversas}

Bartlect (cit. 24) habla de incisión longitudinal de piel, separación de rectos y apertura de fascia y peritoneo en sentido transversai. Kustner, Segond, etc. (cit. 20), hablan de incisión de piel transversal y vertical de línea blanca con todos sus elemen. tos así comu del peritoneo. Otros autores (4) mencionan diversas incisiones transversas para abdomen superior ocasionalmente aplicables a abdomen inferior. Ninguno do estos tipos de incisión gozan de popularidad en la actualidad en cirugía ginecológica y obstétrica y no encontré referencia a ellas en las publicaciones recientes corisultadas.

\section{Consideraciones estadísticas}

Se presentan las estadisticas de varios autores quienes comparan los resultados de las incisiones transversas y longitudinales según su experiencia en varios centros hospitalarios.

Singleton (cit. 13), en 3.147 incisiones transversas halla:

Eviceración post-operatoria inmediata .. . . $0.031 \%$ Eventraciones tardías . . . . . . . . . . . . . . . $0.92 \%$

En 6.000 incisiones longitudinales:

Eviceración post-operatoria inmediata ...... $1 \%$

Eventraciones tardías . . . . . . . . . . . . . $2,2 \%$

Farris (cit. 15) encontró que las evisceraciones son cinco veces más frecuentes en las incisiones longitudinales que en las 
transversales y que las eventraciones son dos y media veces más frecuentes.

Rees y Coller (cit. 15) informaron sobre las complicaciones pulmonares, las culales se presentan en un $2,6 \%$ en las incisiones transversas y un $9,5 \%$ en las verticales

Alvarez Bravo refiere que ha practicado una serie de 169 ope. raciones ginecológicas por la incisión de Pfannenistiel Rodriguez, desde extirpación de pequeños tumores ováricos hasta tipo Wertheim y similares, así como cesáreas, con solamente cuatro complicaciones: 1 hematoma subcutáneo y 3 casos de infección de la herida.

En una serie muy bien controlada del Lovisville General Hospital Ky., Departamento de Ginecología, durante los años de 1951 y 1952 se hallaron los siguientes resultados (25):

\section{Porcentaje de diversos tipos de laparotomías en el "Louisville General Hospital”}

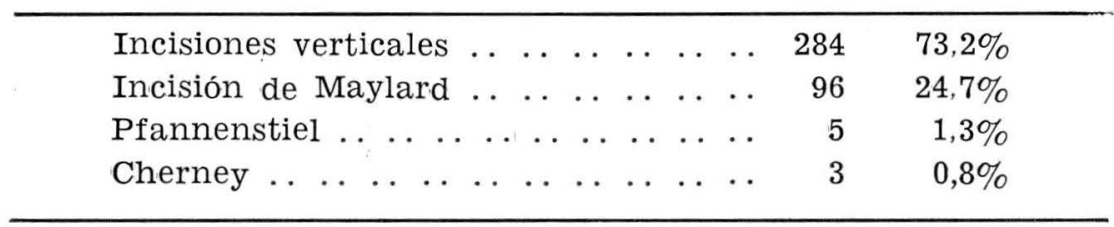

En este cuadro vemos claramente que aún se da gran preferencia a la incisión vertical para toda clase de intervenciones; y que de las incisiones transversas, la que más aceptación ha tenico es el Maylard. La mortalidad hallada en esta serie es de 1,04\% y no es directamente imputable a la incisión, pues se trataba de una paciente que muere en post-operatorio por adenocarcinoma ciel cuerpo uterino con metástasis.

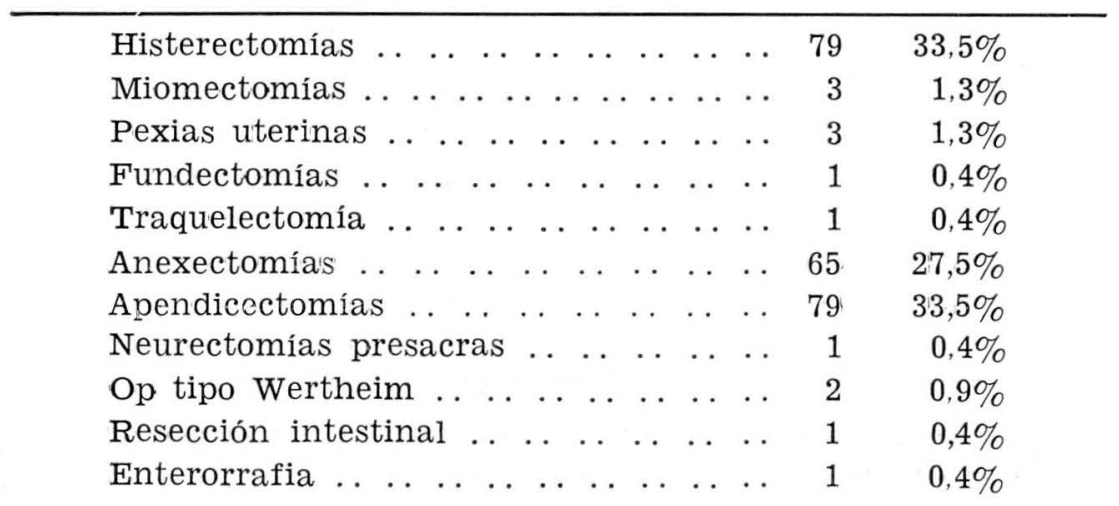


Intervenciones quirúrgicas realizadas por incisiones transversas en el "Lousville Hospital" en la serie estudiada.

Se presentaron las siguientes complicaciones:

Complicaciones de la serie anterior

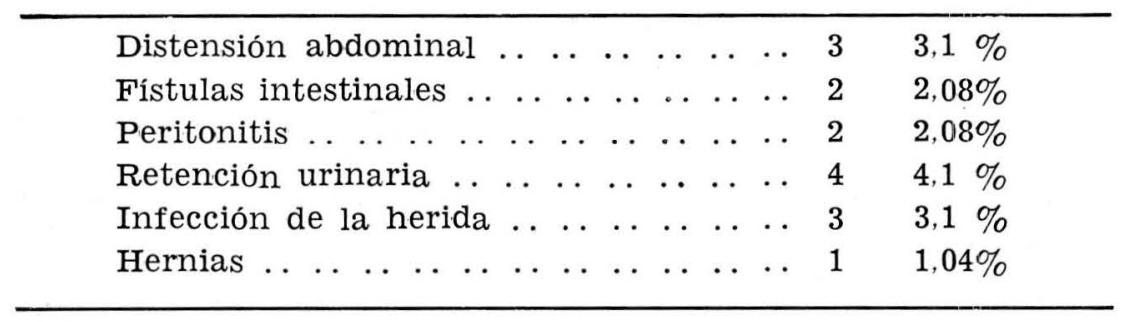

De las hernias quirúrgicas que se presentaron el 1951 y 1952 se halló la siguiente proporción:

Hernias post-operatorias de toda la serie anterior

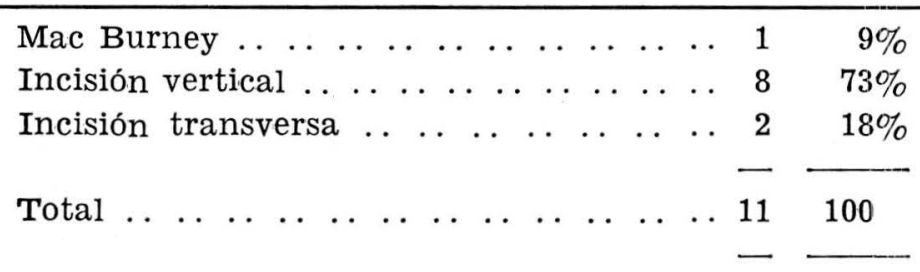

En una serie de 26 casos el doctor Durán (7) halló los siguientes valores estadísticos:

Tipos de intervención realizadas por la incisión de Maylard

$\begin{array}{lllllllllllll}\text { Histerectomía total } \ldots & \ldots & \ldots & \ldots & \ldots & \ldots & \ldots & \ldots & \ldots & \ldots & 8 \\ \text { Op tipo Wertheim y similares } & \ldots & \ldots & \ldots & \ldots & \ldots & \ldots & 3 \\ \text { Anexectomías } \ldots & \ldots & \ldots & \ldots & \ldots & \ldots & \ldots & \ldots & \ldots & \ldots & \ldots & \ldots & 10 \\ \text { Miomectomía de histeropexias } & \ldots & \ldots & \ldots & \ldots & \ldots & \ldots & 1 \\ \text { Salpingolosis y apendicectomías } & \ldots & \ldots & \ldots & \ldots & \ldots & 1 \\ \text { Operación cesárea } \ldots & \ldots & \ldots & \ldots & \ldots & \ldots & \ldots & \ldots & \ldots & \ldots & \ldots & 3\end{array}$

Las complicaciones halladas en este grupo de pacientes fueron las siguientes: 


\section{Complicaciones de la serie anterior}

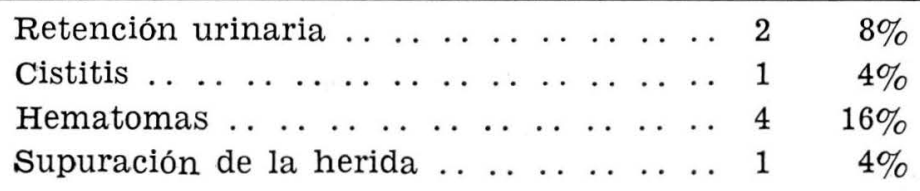

En una serie (2) de laparatomías tipo Pfannenstiel de su cientela particular, por lo cual fueron controladas más cuidadosamente por el mismo cirujano, se obtuvieron los siguientes resultados: se realizaron operaciones tan variadas como 6 histerectomías, 2 anexectomías, 3 apendicectomías, 3 salpingectomías, 3 plastías tubarias, 1 pexia uterina, 14 resecciones parciales de cvario uni o bilaterales, 4 extirpaciones de quistes ováricos, 3 neurectomías presacras, 1 operaciones de Estes, 2 aislamientos de trompas, 1 extirpación de quiste de la hidatide torcido y 2 cesáreas. Solo se hallo una complicación: hematoma subcutáneo que se drenó insertando una pinza por la línea de sutura y que curó sin complicaciones ulteriores.

El mismo autor en una serie total de 150 casos, incluyendo los anteriores, solo tiene 3 complicaciones: 2 hematomas, 1 infección, Como vemos, esta larga serie de casos es comparable a la presentada por Alvares, lo cual nos indica que tanto el Pfannenstiel original como el modificado, son igualmente ventajosos si se practican con la técnica adecuada y permiten toda clase de cirugía ginecológica y obstétrica; a pesar de las objeciones que se le hacen a esta incisión.

En la mayor serie estadística muestra (14) realizadas sobre un total de 173 laparatomías realizadas en el año de 1958 en el Departamento de Ginecología del Hospital San Juan de Dios, D. E., ce Bogotá $\left({ }^{*}\right)$, hallamos los siguientes datos:

(*) Algunas pocas intervenciones de tipo urológico se practicaron en el Departamento de Urología, contíguo al de Ginecología y en colaboración con éste. 


\section{Cuadro I}

Tipos de laparotomía

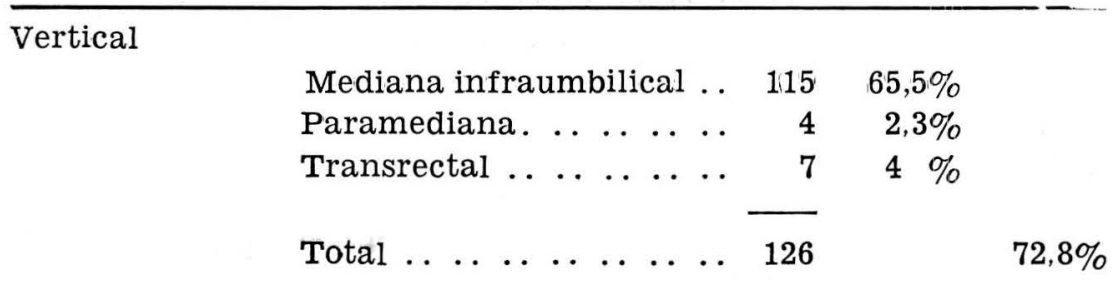

Transversal

\begin{tabular}{|c|c|c|}
\hline Pfannenstiel típico . . & 16 & $9,2 \%$ \\
\hline Pfannenstiel modificado.. & 19 & $11 \%$ \\
\hline Mac Burney ......... & $\begin{array}{r}2 \\
-\end{array}$ & $1,2 \%$ \\
\hline
\end{tabular}

No descritas en hoja quirúrgica $\ldots \ldots \ldots \ldots$. . $10 \quad 5,8 \% \quad 5,8 \%$ $\begin{array}{lllllllllllllllllllllll}\text { Totales } & \ldots & \ldots & \ldots & \ldots & \ldots & \ldots & \ldots & \ldots & \ldots & \ldots & \ldots & \ldots & 173 & 100 & \% & 100 & \%\end{array}$

Tipos de incisiones realizadas en el Departamento de Ginecología del Hospital de San Juan de Dios, en 1958.

Por estas incisiones se practicaron una o más intervenciones a la vez, las cuales detallamos a continuación; el total fue de 237 intervenciones. Véase cuadro número 2: Intervenciones realizadas.

Las complicaciones y mortalidad que encontramos en esta serie se presentan a continuación en el cuadro número 3: Complicaciones y mortalidad de una serie de 163 laparotomías: 126 verticales y 37 transversales. En él se comparan el porcentaje de complicaciones en las incisiones verticales y en las transversales, simultáneamente. 


\section{Cuadro II}

\section{Intervenciones realizadas}

\begin{tabular}{|c|c|c|}
\hline Histerectomía total $\ldots \ldots \ldots \ldots \ldots \ldots$ & 61 & $25,8 \%$ \\
\hline Histerectomía subtotal .. . . . . . . . & 0 & - \\
\hline Wertheim y similares ........... & 6 & $2,5 \%$ \\
\hline Resecciones ganglionares $\ldots \ldots \ldots \ldots$ & 1 & $0,4 \%$ \\
\hline $\begin{array}{llllllllll}\text { Miomectomías } & . & \ldots & \ldots & \ldots & \ldots & \ldots & \ldots & \ldots\end{array}$ & 11 & $4,6 \%$ \\
\hline Pexias uterinas $\ldots \ldots \ldots \ldots \ldots \ldots \ldots$ & 1 & $0,4 \%$ \\
\hline $\begin{array}{c}\text { Embarazos no diagnosticados, solo se } \\
\text { practicó la laparatomía ........ }\end{array}$ & 2 & $0.8 \%$ \\
\hline $\begin{array}{llllllll}\text { Uterosigmoidostomia } & \ldots & \ldots & \ldots & \ldots & \ldots & \ldots\end{array}$ & 1 & $0,4 \%$ \\
\hline Cistectomía parcial . . . . . . . . . . & 1 & $0,4 \%$ \\
\hline Ureteroneosistostomía $\ldots \ldots \ldots \ldots$ & 1 & $0,4 \%$ \\
\hline Fistulorrafia vesical (vía abdominal) .. & 1 & $0,4 \%$ \\
\hline Anexectomía $\ldots \quad \ldots \ldots \ldots \ldots \ldots \ldots$ & 82 & $34,7 \%$ \\
\hline $\begin{array}{lllllllllll}\text { Salpingolisis } & \ldots & \ldots & \ldots & \ldots & \ldots & \ldots & \ldots & \ldots & \ldots\end{array}$ & 1 & $0,4 \%$ \\
\hline Salpingectomía $\ldots \ldots \ldots \ldots$ & 11 & $4,6 \%$ \\
\hline $\begin{array}{llllllll} & & & & & & & \\
\end{array}$ & 5 & $2,1 \%$ \\
\hline 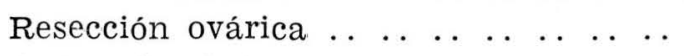 & 9 & $3,9 \%$ \\
\hline Ooforectomía $\ldots \ldots \ldots \ldots \ldots \ldots \ldots$ & 6 & $2,5 \%$ \\
\hline Drenaje absceso apendicular ....... & 1 & $0,4 \%$ \\
\hline Apendicectomías $\ldots \ldots \ldots \ldots \ldots \ldots$ & 27 & $11,5 \%$ \\
\hline Suturas intestinales $\ldots \ldots \ldots \ldots \ldots \ldots$ & 1 & $0,4 \%$ \\
\hline Resecciones intestinales $\ldots \ldots \ldots \ldots$ & 1 & $0,4 \%$ \\
\hline Herniorrafias $\ldots \ldots \ldots \ldots \ldots \ldots \ldots$ & 7 & $3 \%$ \\
\hline$\ldots$ & 237 & $100 \%$ \\
\hline
\end{tabular}

NOTA.-Complicaciones y mortalidad comparativa halladas cntre las incisiones verticales y las transversales en el Departamento de Ginecología, se analizan en el cuadro siguiente, número 3. 


\section{Cuadro III}

Complicaciones y mortalidad en una serie de 163 laparotomías (126 verticales y $3 y$ transversales)

\begin{tabular}{|c|c|c|c|c|}
\hline & \multicolumn{2}{|c|}{$\begin{array}{l}\text { En una se- } \\
\text { rie de } 126 \text { in- } \\
\text { cisiones ver- } \\
\text { ticales }\end{array}$} & \multicolumn{2}{|c|}{$\begin{array}{l}\text { En una se- } \\
\text { rie de } 37 \text { in- } \\
\quad \text { siciones } \\
\text { transversales }\end{array}$} \\
\hline Neumonía . . . . . . . . . & 2 & $1,6 \%$ & 0 & - \\
\hline Bronquitis leve $\ldots \ldots \ldots$ & 3 & $2,4 \%$ & 1 & $2,7 \%$ \\
\hline Tromboflebitis $\ldots \ldots \ldots$ & 0 & 一 & 1 & $2,7 \%$ \\
\hline Distensión abdomnial . . . & 21 & $16,8 \%$ & 0 & - \\
\hline Fístulas intestinales ... . & 1 & $0,8 \%$ & 0 & - \\
\hline Peritonitis $\ldots \ldots \ldots \ldots$ & 1 & $0,8 \%$ & 0 & - \\
\hline Retención urinaria ...... & 5 & $4 \%$ & 0 & - \\
\hline Hematomas $\ldots$. . & 9 & $7,2 \%$ & 2 & $5,4 \%$ \\
\hline Infección-incisión $\ldots \ldots$ & 25 & $20,0 \%$ & 3 & $8,1 \%$ \\
\hline Eviseración $\ldots \begin{array}{lllll} & \ldots & \ldots & \ldots & \ldots\end{array}$ & 3 & $2,4 \%$ & 0 & - \\
\hline $\begin{array}{l}\text { Oclusión intestinal por ad- } \\
\text { herencias } \ldots \\
\ldots\end{array}$ & 1 & $0,8 \%$ & 0 & - \\
\hline Abscesos cúpula vaginal .. & 11 & $8,8 \%$ & 2 & $5,4 \%$ \\
\hline Vómito $\ldots \ldots \ldots \ldots$ & 14 & $11,2 \%$ & 1 & $2,7 \%$ \\
\hline Incontinencia de orina. . & 1 & $0,8 \%$ & 0 & - \\
\hline Fístula urinaria $\ldots \ldots \ldots$ & 3 & $2,4 \%$ & 0 & - \\
\hline 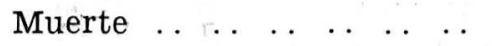 & 1 & $0,8 \%$ & 0 & - \\
\hline
\end{tabular}

Complicaciones y mortalidad comparativa halladas entre las incisiones verticales y las transversales en el Departamento de Ginecología del Hospital de San Juan de Dios.

La causa de muerte de la única defunción que se presentó en el servicio durante el año de 1958, fue, según autopsia número 0362-58 del 20 de noviembre de 1958, Profundos trastornos neurovegetativos".

Ventajas e inconvenientes de las incisiones transversas

Toda buena incisión en cirugía debe llenar los siguientes requisitos:

Dar campo adecuado. 
Respetar las estructuras anatómicas en lo posible.

Ser fácil de prolongar.

Obtener buenos efectos estéticos.

No se ha encontrado la incisión que llene completamente los requisitos mencionados, pero varias se acercan a esto.

$\mathrm{La}$ incisión transversal tiene las siguientes ventajas e inconvenientes:

Mejor campo operatorio: se cae más directamente sobre los ćrganos pélvicos objeto de la intervención, y recuérdese que la incisión mediana puede prolongarse hacia arriba, pero que cada vez ros alejamos más de la pélvis, la distancia umbílico-pubiana es un $25 \%$ más corta que la biespino-ilíaca-anterior y superior.

En las personas obesas es fácil rechazar el grueso delantal de abdomen péndulo y hacer la incisión por debajo, con lo cual los bordes de la incisión son más delgados.

Respecto al hecho de no poder hacer exploraciones en abdomen superior, recuérdese que "dos buenas incisiones son mejores que una mala" (Williams, 25).

El cierre de la incisión en los casos difíciles con el paciente anestesiado superficialmente y pujando, se facilita flejando ligeramente la mesa y debido a la dirección de los cortes casi no se presenta el problema de la aproximación de los diferentes planos.

El tiempo de cierre de la incisión de Pfannenstiel, Pfannestiel, Rodríguez, Maylard, es prácticamente igual al empleado para el cierre de las incisiones verticales.

Por las razones antes expuestas, por la mejor irrigación, por no alterarse casi la inervación, por no ejercerse tracción sobre la línea de sutura que es paralela a las fibras musculares, se obtienen mejores cicatrices.

Se produce menos dolor en el post-operatorio por haber menos filetes nerviosos seccionados y por esto mismo se presentan menos complicaciones post-operatorias de tipo respiratorio por la buena ventilación pulmonar.

En caso de presentarse infección, ésta es fácilmente combatida por la buena irrigación, y si es necesario practicar drenaje, se obtiene rápida evacuación de la colección purulenta por poder hacerlo en la parte más declive, lo cual es muy difícil de lograrlo en las incisiones longitudinales con el enfermo en decúbito dorsal. 
Raras veces se presentan adherencias post-operatorias, pues como lo han demostrado Mc.Arthur, Moore en repetidas autopsias (citado 13) la tensión sobre el peritoneo las favorece y aquí la tensión es casi nula (25).

Los resultados estéticos son óptimos, pues estas incisiones cicatrizan mejor por haberse lesionado menos elementos de la pared abdominal, y además son ocultadas en parte por el vello pubiano y los pliegues naturales de la región.

Desde el punto de vista de problemas anestésicos es necesario menos Trendelenburg, con lo cual el paciente respira mejor y expuesto a menores cambios hemodinámicos durante el acto opeustá expuesto a menores cambios hemodinámicos durante el acto operatorio.

Si se emplea anestesia raquídea baja y Trendelenburg, acentuado asi como maniobras intra-abdominales bruscas y grandes rollos para lograr una buena exposición pélvica, el paciente acusa dolor y presenta cambios tensionales y aún shok; todo esto se evita por las razones antes vistas y recordando que casi siempre es suficiente una compresa común para rechazar las vísceras.

Las complicaciones y la morbilidad son casi nulas, como to prueban todas las estadísticas consultadas y en lo que están de acuerdo la mayoría de los autores.

Se ha dicho que es más laboriosa de ejecutar la incisión transversa y que por lo tanto, se gasta más tiempo en llegar a la lesión, lo cual no la hace apropiada para casos de urgencias ginecológicas, como un embarazo ectópico-roto, pero podemos escoger entre los varios tipos de incisión transversa, la que más convenga y en este caso usaremos el Pfannesntiel Rodríguez que se puede ejecutar en menos de 2 ininutos y en el que prácticamente no necesita hacerse hemostacia con pinzas, como lo ha comprobado Grenhill (21) observando operar al doctor Rodríguez.

Respecto a la falta de buen campo operatorio, las incisiones transversales modernas proporcionan un campo adecuado por la apertura de la aponeurosis de los músculos anchos y la divulsión de sus fibras y el corte de los rectos, ya sea según la técnica de Maylard o Cherney y la apertura transversal del peritoneo y iascia posterior; además, al caer directamente sobre los órganos pélvicos y no ser casi molestado por las asas intestinales, con el uso de separadores adecuados tipo O'Sullivan-O'Connor y similares como por ejemplo: 2 separadores de Gosset colocados uno a la derecha y otro a la izquierda (y no los comunes para laparotomía vertical) si se usa la incisión con corte de rectos, per- 
miten hacer las mayores intervenciones ginecológicas u obstétricas como Werthein o similares, cesáreas (28), (27), etc., con toda facilidad, desde el punto de vista del buen campo operatorio.

Es muy importante recordar que se pueden combinar dos tipos de incisión transversal si es necesario, como por ejemplo, ૯] Pfannenstiel-Rodríguez con el Maylard, etc.

\section{Resumen y conclusiones}

Se hace una breve reseña histórica del tema.

Se describen varias técnicas y sus modificaciones.

Se presentan estadísticas extranjeras y nacionales sobre el tema, en las cuales vemos que todas concuerdan en la bondad de las incisiones transversas y la baja incidencia de complicaciones comparada con las de las verticales.

Se analizan las ventajas y desventajas de las incisiones transversas, mostrando que prácticamente en todos los casos de cirugía obstétrica o ginecológica se puede usar una incisión transversa, adecuada que da buen campo operatorio y está casi libre de complicaciones.

\section{Bibliografía}

1. ALVAREZ BRAVO.-Incisión transversa; Ginecología y Obstetricia de México (Septiembre - Octubre de 1951. Páginas 297 y siguientes).

2. AMOROCHO CARREÑO JORGE.-Comunicaciones personales y Estadísticas en Clientela Privada.

3. CURRENT LIST OF MEDICAL LITERAT'URA.-Años 1952, 53, 54, 55, 56 y 58.

4. CHRISMAN-OTTOLENGHI.-Raffo Von Grolman. Técnica Quirúrgica. 7?a Edición. El Ateneo. Buenos Aires. Páginas 14-59 y siguientes.

5. DELANY J. J. STEPHENS W. W.-Incisión transverse. Texas State Journal of Medicine. Feb. 52. Pág. 8g a 93.

6. DEXEU' FONT.-Tratado de Obstetricia. Tomo II. Páginas $1.441 \mathrm{y}$ siguientes. Salvat. Editores, S. A., 1957. Barcelona.

7. V. FABIO DURAN VELAZCO.--Memorias de la II Convención Colombiana de Obstetricia y Ginecología. Páginas 331 y siguientes. Editada "Revista Colombiana de Obstetricia y Ginecología".

8. EXCERPTA MEDICA.-Volumen I a volumen IX (1948 a 1956).

9. FINOCHIETO R. VASQUEZ M. J.-La incisión de Cherney. Prensa Médica, Argentina, 8 de mayo de 1953. 40:19. Páginas 1.129 a 1.135.

10. FERRARIS F.-Pubic Minimal Incisión for gy. surgeri. Minerva Ginecológica. Torino. Agosto, 1952. 1:9. Páginas 355 a 360.

11. GAUDRI.-Inciscion transversa. Canadian Hospital. Toronto, noviembre 51. 28:1. Páginas 32 a 34 .

12. GEILER L. J.-Incision transverse Khirurgia. (Mosawa). Número 2. Febrero, 1952. Páginas 56 a 64 .

13. DANIEL GOMEZ.-Incisi6n Transversa. Prensa Médica. 44: 49. 3.521. 1957. 
14. GOUBERT CARLOS A.-Estadísticas del Departamento de Ginecologia del Hospital de San Juan de Dios. 1958. Revista Facultad de Medicina de la Universidad Nacional". (En prensa).

15. J. P. GREENHILL B. S. M. D. FACS.-Tranverse incision in pelvic surgery. Year boock of Obstetrics and Gynecology, 1955-56. Páginas 309 y 210.

16. MARECK C. B. DOLCE J. R.-Incision Lower midline ternic and advantages. Marylad State Medical Journal (Baltimore). Septiembre, 1952. Páginas 447 a 452 .

17. KOZLOWSK POLSKI PREGLAD.-Tranverse incisión complications adhesion post-op. Cirurgiczny. Warszawa. Mayo, 1952. Páginas 194 a 197.

18. KNOBLAVEH H., BETZIER H. J.-Pararrectal sliding for transverse peritoneal section. Cirug (Berlim). Noviembre, 1952. 23:11. Páginas 519 y 520.

19. KRAPPE.-Transrectal incisión with differ direction in gall blader surg. Zentrablatt fur Ciruge (Leizip). 77:39. 1952.

20. R. PROUST.-Aparato genital de la mujer. Páginas 197 y siguientes. Editorial Nacional. México, 1950.

21. RODRIGUEZ LOPEZ.-Rápida incisión de Pfannenstiel. Year Book of Obstetric and Ginecology, 1948. Páginas 443 y 444.

22. SUTLEW M. R.-Incision Transverse. Journal of the National Medical Association (New York). Mayo, 1952. Páginas 133 y 134.

23. TE LINDE RICHARD.-Ginecología Operatoria. 2a Edición. Editorial José Bermúdez. Buenos Aires. Página 45.

24. TOREK.-Técnica Quirúrgica. Tomo III. Salvat. Editores, S. A., 1953. $2^{\text {a }}$ Edición. Barcelona. Páginas 1.707 y siguientes.

25. WILLAMS G. S. LOW.-Transverse with incisión of rectus muscle American Journal of Obs. and Gyn. (Sant Louis). Febrero, 1954. 67:2. Páginas 398 a 406.

26. RUSEEL WEITP.-(Cit. Greenhill Y. B. of O. And G. 1955-56) .

27. CALCANO JULIO.-Incisión de Pfannestiel modificada en cesáreas. Reimpreso de la "Revista de Obstetricia y Ginecología". Venezuela. "Revista Colombiana de Obstetricia y Ginecología". Septiembre-octubre, 1954. Volumen IV. Número 1. Página 66.

28. CASTILLO VEGA EUDORO.-Incisión de Pfannestiel en la Cesárea. "Revista Colombiana de Obstetricia y Ginecología". Volumen V. Número 2. Octubre-noviembre, 1953. 